\title{
Optimizing Azadi Controller with COA
}

\author{
Ashkan Aghaei \\ Department of Electrical Engineering \\ Semnan University, Semnan, Iran
}

\author{
Sassan Azadi \\ Department of Electrical Engineering \\ Semnan University, Semnan, Iran
}

\begin{abstract}
Cuckoo Optimization Algorithm (COA) is one of the hottest meta-heuristic algorithms. Finding the best optimal point, rapid convergence, simplicity in determining algorithm parameters are some merits of COA.

Azadi controller is one of latest method of adaptive controlling. It is simple, robust, effective and immune against noise and plant's variations. All of them make it unique and without no compotator. To tune it, there are three parameters. On this paper, COA undertakes responsibility of tuning these parameters to achieve the best response.
\end{abstract}

Catalytic Continuous Stirred Tank Reactor (CSTR) is an ordinary industrial system and it is a decent example to survey Azadi controller that is designed by COA.

\section{General Terms}

Adaptive control, Evolutionary Algorithm.

\section{Keywords}

Azadi controller, COA, CSTR.

\section{INTRODUCTION}

Azadi controller is a novel adaptive controller. It is one of latest method of adaptive controlling. It is simple, robust, effective and immune against noise and plant's variations. All of them make it unique and without no compotator. To tune it, like PID controller there are three parameters $\alpha_{0}, \alpha_{1}$, and $\alpha_{2}$. There are some tips to tune it [1] [2] [3] [4] [5] but is time consuming and in some situation, achieving to the best selection is bothersome. To solve this difficulty, utilizing meta-heuristic algorithms for finding the optimal point is recommended [6].

In the last few years, several attempts have been made to synthesize the desired control system such as Fuzzy Logic System (FLC) by learning or optimization methods. The most important part of the self-tuning systems is the online tuning algorithm [7] [8] [9]. The gradient descent algorithm [10] and genetic algorithm (GA) [11] [12] [13] [14] [15] [16] have been used as the base for the learning process. Simulated annealing (SA) [17] [18] [19] is yet another means of solving optimization problems that has slowly gained interest of researchers for learning and tuning of control systems. Particle swarm algorithm is the other way for optimizing control systems which is introduced later than the others [20] [21] [22] [23].

The ability of COA to Finding the best optimal point, rapid convergence, simplicity in determining algorithm parameters and to handle any type of cost function for large scale problems makes it a better tool than the traditional gradient descent algorithm, Genetic algorithm (GA) and Particle swarm algorithm.

COA is one of the hottest meta-heuristic algorithms. Finding the best optimal point, rapid convergence, simplicity in determining algorithm parameters are some merits of COA.
All of them prove the idea that the best option for tuning parameters of Azadi controller is the COA. Without no cast of doubt, combination of these both recent and robust method leads to one unique and operational style of control technique. It is a breakthrough in control knowledge. Inasmuch as it is an intelligent control without no calibration and tuning, control system could tune itself independently and be immune against noise and plant's variations. Maybe in near future all control system in all field of human life would be such as this way.

Catalytic Continuous Stirred Tank Reactor (CSTR) is an ordinary industrial system and it is a decent example to survey this issue.

\section{CUCKOO OPTIMIZATION ALGORITHM (COA)}

Inasmuch as the COA has been published recently. It seems brief introduction is inescapable. COA is inspired by the life of a bird family which is called Cuckoo. Special lifestyle of these birds and their characteristics in egg laying and breeding has been the basic motivation for development of this new evolutionary optimization algorithm. The cuckoo population, in different societies, is in two types: mature cuckoos and eggs. The effort to survive among cuckoos constitutes the basis of COA. During the survival competition some of the cuckoos or their eggs, demise. The survived cuckoo societies immigrate to a better environment and start reproducing and laying eggs. Cuckoos' survival effort hopefully converges to a state that there is only one cuckoo society, all with the same profit values. Figure 1 depicts flowchart of COA.

Similar to other evolutionary methods, COA starts with an initial population. These initial cuckoos have some eggs to lay in some host birds' nests. Some of these eggs which are more similar to the most bird's eggs have the opportunity to grow up and become a mature cuckoo. Other eggs are detected by host birds and are killed. The grown eggs reveal the suitability of the nests in that area. The more eggs survive in an area, the more profit is gained in that area. Hence, the position in which more eggs survive will be the term that COA is going to optimize. Cuckoos search for the most suitable area to lay eggs in order to maximize their eggs survival rate. After remained eggs grow and turn into a mature cuckoo, they make some societies. Each society has its habitat region to live in. The best habitat of all societies will be the destination for the cuckoos in other societies. Considering the number of eggs each cuckoo has and also the cuckoo's distance to the goal point (best habitat), some egg laying radii is dedicated to it. Then they immigrate toward this best habitat. They will inhabit somewhere near the best habitat. Then, cuckoo starts to lay eggs in some random nests inside her egg laying radius. This process continues until the best position with maximum profit value is obtained and most of the cuckoo population is gathered around the same position. For further details, please peruse [24] 


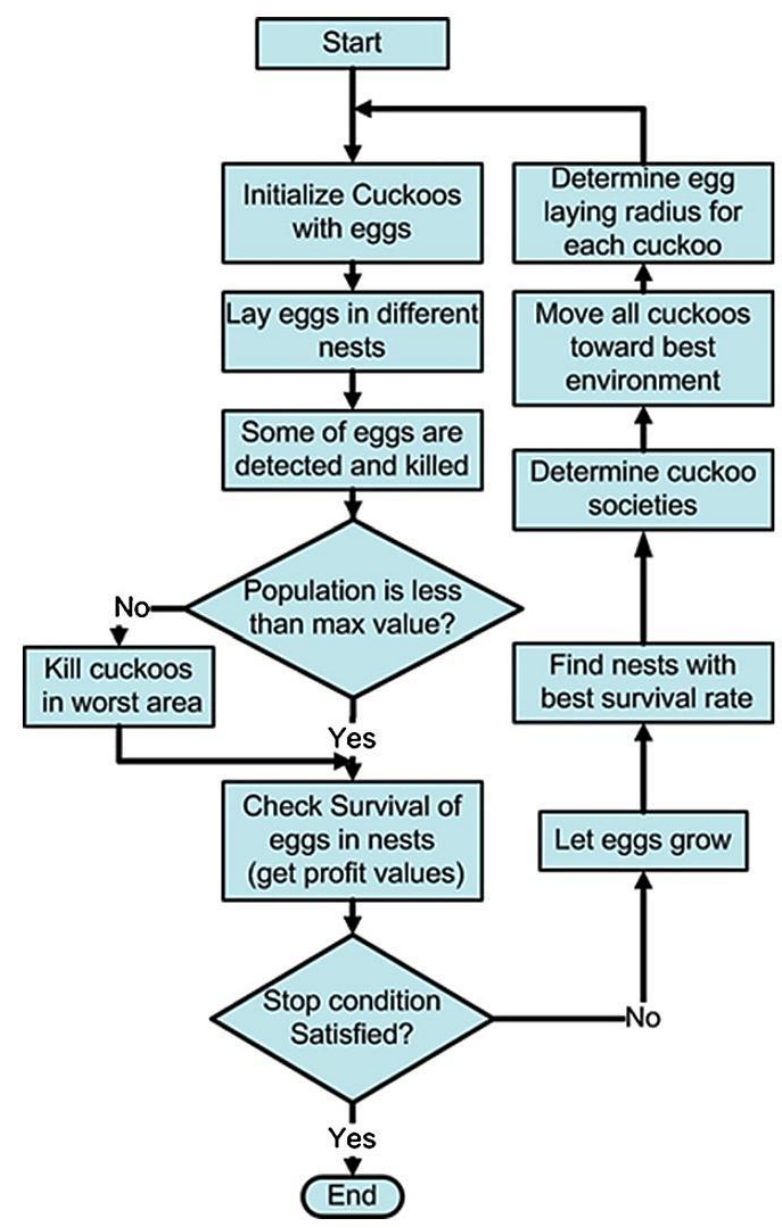

Fig 1: Flowchart of Cuckoo Optimization Algorithm.

\section{AZADI CONTROLLER}

Due to the fact that Azadi controller has been available lately, brief summarizing about this method of control is inevitable.

Azadi controller is a novel adaptive controller. Its idea comes from a neurophysiology phenomenon. That is why the nature always stays at the optimal design point, inspiring this nature makes the controller be simple, and effectively. Modeling of nerve cells action potential inspires idea of Azadi controller that leads to Simulink model that is depicted in figure 2.

$\mathrm{f}(\mathrm{v})$ is function with following equation

$f(v)=\frac{\alpha_{0}-\alpha_{1} v+\alpha_{2} v^{2}}{1+v+v^{2}}$

$v$ in preceding equation is the absolute value of error divided by the derivative of error. Since, these parameters are substantial for the system responses. Based on these two values, the controller should change its output to achieve a decent control.

$v=\left|\frac{e(t)}{e^{\prime}(t)}\right|(2)$

Positive feedback is vital part of Azadi controller. Azadi controller has two negative and one positive feedback. To tune it there are some parameters $\alpha_{0}, \alpha_{1}$, and $\alpha_{2}$. The three parameters play different acts based on the system performances similar to a PID controller. Figure 3 demonstrates a simple response of three values of these parameters.

As shown in figure 3 , the start-up value is $\alpha_{0}$, and the end-up value is $\alpha_{2}$. The positive feedback gain works in the midway region. The positive feedback gain is surrounded by two strong negative feedbacks. Once again, utilizing a positive feedback gain, surrounded by two other negative feedbacks has been inspired from nature [25] [26]. The minimum value of the $f(v)$ function can be found by setting derivative of $f(v)$ to zero, i.e. when:

$$
\begin{aligned}
& \frac{d f(v)}{d v}=0 \\
& v_{0}=\frac{-\left(\alpha_{2}-\alpha_{0}\right)+\sqrt{\Delta}}{\alpha_{2}-\alpha_{0}}
\end{aligned}
$$

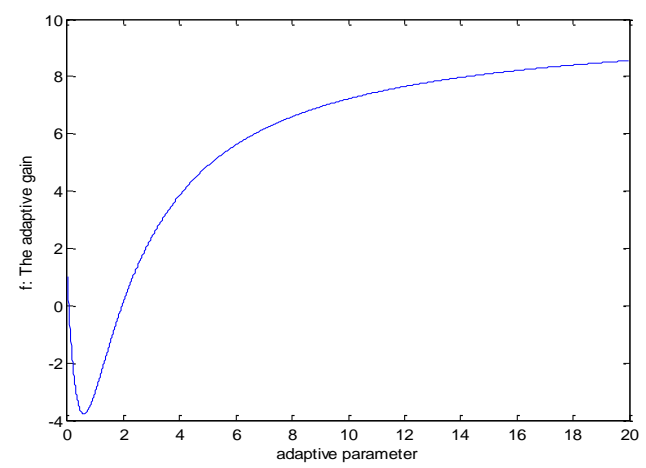

Fig 2: The function $f(v)$ verses $v$ for some arbitrarily values of $\alpha_{0}=1, \alpha_{1}=20$, and $\alpha_{2}=10$. Since the value of $\alpha_{1}$ provide a positive feedback, this function gets a negative value in the vicinity of $v=0$.

In $v_{0}$ which is the $v$ value when $\mathrm{f}(\mathrm{v})$ is minimum. The value of $\Delta$ is:

$\Delta=\alpha_{0}^{2}+\alpha_{1}^{2}+\alpha_{2}^{2}+\alpha_{0} \alpha_{1}+\alpha_{1} \alpha_{2}-\alpha_{0} \alpha_{2}$

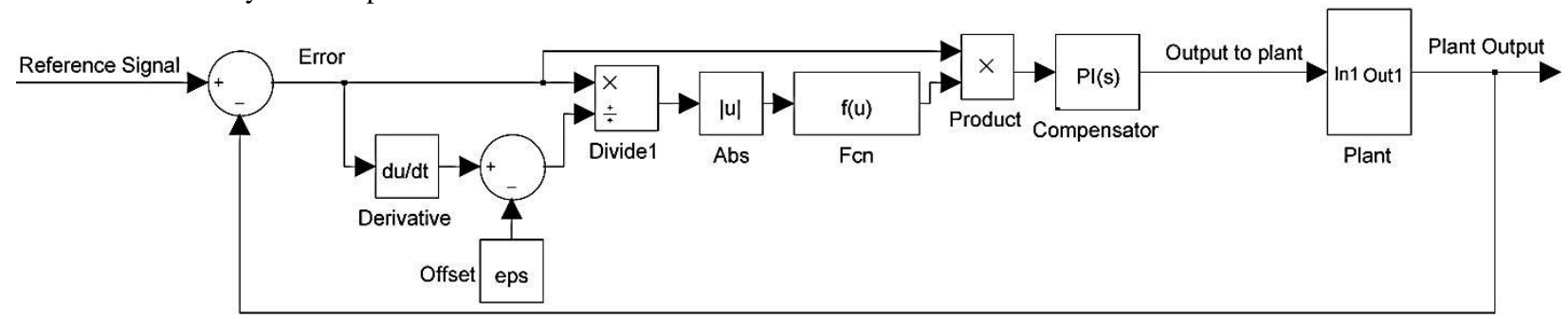

Fig 3: Simulink model of Azadi controller. 
Then the minimum value of $f(v)$ is:

$\operatorname{Min} f(v)=f(v 0)=\frac{\alpha_{0}-\alpha_{1} v_{0}+\alpha_{2} v_{0}^{2}}{1+v_{0}+v_{0}^{2}}$

As can be seen in figure 3 , the value of $f\left(v_{0}\right)$ can be negative (positive feedback). In the design work, the variable gain $\mathrm{f}(\mathrm{v})$ varies from:

$\mathrm{f}\left(\mathrm{v}_{0}\right) \leq \mathrm{f}(\mathrm{v}) \leq \alpha_{2}(7)$

As shown in figure 2, a compensator is added to stabilize the plant for the variable gain of Azadi controller. This compensator can be just an integrator to provide increase the type of plant. A small number like $1^{-10}$ is added to avoid dividing by zero that is indicated by 'offset'. To achieve details regarding Azadi Controller please refer [1] [2] [3] [4] [5].

Tuning Azadi controller with COA is examined by a plant which is a Catalytic Continuous Stirred Tank Reactor (CSTR). In the subsequent section, details of this plant is introduced.

\section{CATALYTIC CONTINUOUS} STIRRED TANK REACTOR (CSTR)

A diagram of the process of CSTR is shown in the following figure 4 .

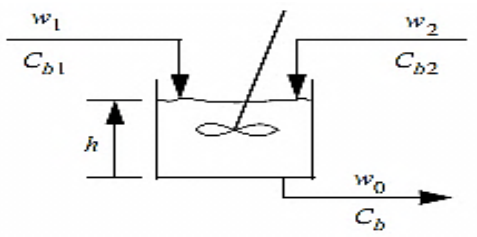

Fig 4: The CSTR diagram Process [27].

The dynamic model of the system is

$$
\begin{aligned}
& \frac{d h(t)}{d t}=W_{1}(t)+W_{2}(t)-0.2 \sqrt{h(t)} \\
& \frac{d C_{b}}{d t}=\left(C_{b 1}-C_{b}\right) \frac{W_{1}}{h}+\left(C_{b 2}-C_{b}\right) \frac{W_{2}}{h}-\frac{k_{1} C_{b}}{\left(1+k_{2} C_{b}\right)^{2}}
\end{aligned}
$$

Where $\mathrm{h}(\mathrm{t})$ is the liquid level, $\mathrm{C}_{\mathrm{b}}(\mathrm{t})$ is the product concentration at the output of the process, $\mathrm{W}_{1}(\mathrm{t})$ is the flow rate of the concentrated feed $\mathrm{C}_{\mathrm{bl}}$, and $\mathrm{W}_{2}(\mathrm{t})$ is the flow rate of the diluted feed $\mathrm{C}_{\mathrm{b} 2}$. The input concentrations are set to $\mathrm{C}_{\mathrm{b} 1}=24.9$ and $\mathrm{C}_{\mathrm{b} 2}=0.1$. The constants associated with the rate of consumption are $\mathrm{k}_{1}=1$ and $\mathrm{k}_{2}=1$.

The objective of the controller is to maintain the product concentration by adjusting the flow $\mathrm{W}_{1}(\mathrm{t})$. To simplify the demonstration, set $\mathrm{W}_{2}(\mathrm{t})=0.1[28]$. The level of the tank $\mathrm{h}(\mathrm{t})$ is not controlled for this experiment. Simulink model of system is presented in figure 5 .

Random signal with 23 units' amplitude and 0.1 variance is added as disturbance noise to control commend each 0.1 second on account of presenting of Azadi control capability to be immune against noise. It is obvious; this disturbance is added after tuning Azadi controller with COA. Furthermore, to prevent applying intolerable gain to plant, Azadi controller output is saturated between 39 to -39 .

The key part of tuning Azadi controller on this paper is designing $\alpha_{0}, \alpha_{1}$, and $\alpha_{2}$ parameters. On next section, method of this task is discussed.

\section{COST FUNCTION AND COA PARAMETERS}

It is taken for granted, each cuckoo position is equal to one style of $\alpha 0, \alpha 1$, and $\alpha 2$ parameters and could be attributed to one system performance. In other words, finding the best cuckoo position yields the best performance, is the target of this paper. In fact, features of flow level response could be as cost function. The criteria for evaluating cost function are rise time (R.T), settling time (S.T), maximum of overshoot (M.O), maximum of undershoot (M.U), and Steady State Error (Ess) of flow level response [29]. It is illustrated in equation (10) and the aim is finding cuckoo position with minimum cost function. This is to say that some weight is intended to each characteristic for changing the influence of each of them.

Cost Function $=\mathrm{W}_{1} \times \mathrm{R} . \mathrm{T}+\mathrm{W}_{2} \times \mathrm{S} . \mathrm{T}+\mathrm{W}_{3} \times \mathrm{M} . \mathrm{O}+\mathrm{W}_{4} \times \mathrm{M} . \mathrm{U}+$ $\mathrm{W}_{5} \times$ Ess

Regarding greater details of COA parameters please refer [24]. In table below is mentioned the parameters of COA.

Table 1. COA parameters.

\begin{tabular}{|c|c|}
\hline COA Parameters & Value \\
\hline Optimization variables & 3 \\
\hline Initial population & 5 \\
\hline Minimum number of eggs laying & 2 \\
\hline Maximum number of eggs laying & 9 \\
\hline Maximum iterations & 15 \\
\hline Number of clusters & 1 \\
\hline Lambda variable & 1 \\
\hline Accuracy & 150 \\
\hline Maximum number of cuckoos & 10 \\
\hline Parameter of egg laying & 1 \\
\hline Population variance & $1^{-10}$ \\
\hline
\end{tabular}

\section{RESULTS}

The COA is run 50 times and following results repeat more than the others. Rates of designed parameters that is associated with the best cuckoo position are $\alpha_{0}=100, \alpha_{1}=504$, and $\alpha_{2}=196$.

Flow level response for $\mathrm{W}_{1}=10, \mathrm{~W}_{2}=10, \mathrm{~W}_{3}=10, \mathrm{~W}_{4}=10$, $\mathrm{W}_{5}=100$ is represented in figure 6 .

The characteristics of this response are identified in table two which breed cost function $=125.5702$.

The COA could tune up Azadi controller parameters promptly with only in six iterations. Besides, level of accuracy is the highest. It is incredible. Figure 7 illustrates amount of cost function is each iteration. 


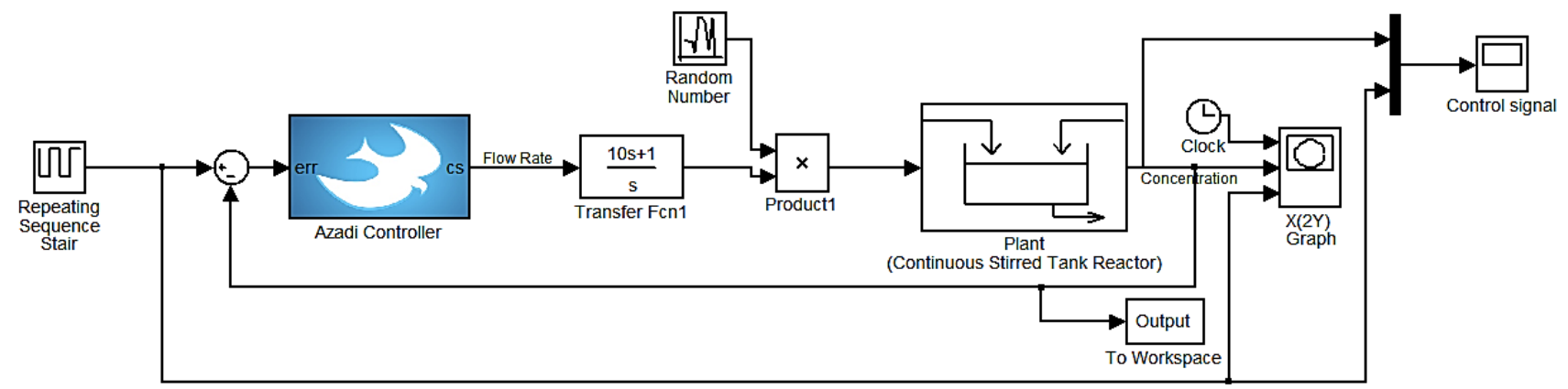

Fig 5: Simulink model of system.

This is worthwhile to mention that all simulation was done by Azadi and fuzzy logic Matlab toolboxes [27].

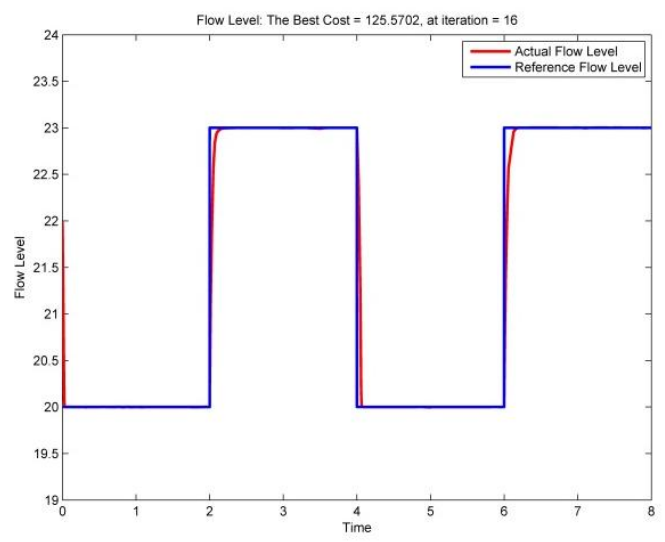

Fig 6: Response of Azadi controller tuned by COA.

Table 2. Characteristics of response.

\begin{tabular}{|c|c|c|c|c|}
\hline Characteristics & $\begin{array}{c}\text { 0 to 2 } \\
\text { Seconds }\end{array}$ & $\begin{array}{c}\mathbf{2} \text { to 4 } \\
\text { Seconds }\end{array}$ & $\begin{array}{c}\mathbf{4} \text { to 6 } \\
\text { Seconds }\end{array}$ & $\begin{array}{c}\text { 6 to 8 } \\
\text { Seconds }\end{array}$ \\
\hline Rise time & 0.018 & 0.056 & 0.044 & 0.079 \\
\hline Settling time & 0.026 & 2.094 & 4.061 & 6.126 \\
\hline $\begin{array}{c}\text { maximum of } \\
\text { overshoot }\end{array}$ & 0.007 & 0.004 & 0.009 & 0.012 \\
\hline $\begin{array}{c}\text { maximum of } \\
\text { undershoot }\end{array}$ & 0 & 0 & 0 & 0 \\
\hline $\begin{array}{c}\text { Steady state } \\
\text { error (ESS) }\end{array}$ & $7.17 \times 10^{-4}$ & $7.75 \times 10^{-4}$ & $3.47 \times 10^{-4}$ & $2.63 \times 10^{-4}$ \\
\hline
\end{tabular}

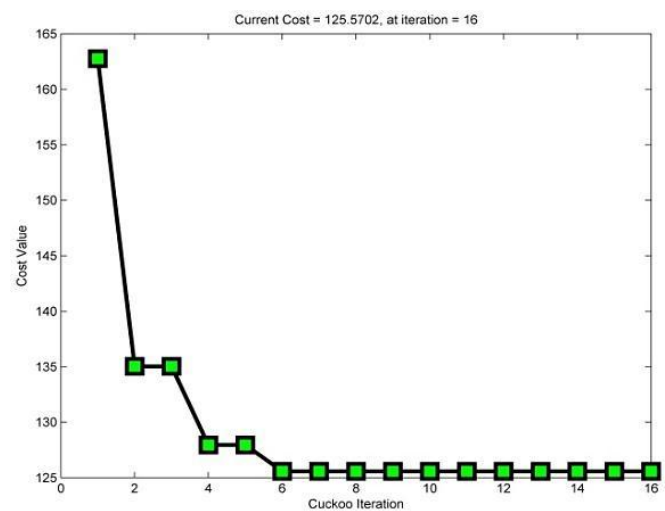

Fig 7: COA iterations.

\section{CONCLUSION}

As expected, on one hand, cuckoo optimization algorithm (COA) could demonstrate its capability to tune up Azadi controller parameters promptly with uppermost level of accuracy. On the other hand, Azadi controller could prove its competence to be immune against noise.

Combination of Azadi controller with COA could control Catalytic Continuous Stirred Tank Reactor (CSTR) and results appear its authority. In the final analysis, this hybrid control system is a breakthrough in control knowledge. Inasmuch as it is an intelligent control without no calibration and tuning, control system could tune itself independently and be immune against noise and plant's variations. Maybe in near future all control systems in all fields of human life would be such as this way.

\section{BIOGRAPHY}

Ashkan Aghaei was born in Isfahan, Iran on September $10^{\text {th }}$ 1984. He received his B.S. degree in Electronics Engineering in 2006. He is student of M.S degree in Mechatronics Engineering in Semnan University.

\section{REFERENCES}

[1] S. Azadi, A. Aghaei and M. A. Hajimousa, "Comparing Azadi Controller with Several Optimal Controllers", Journal of Basic and Applied Scientific Research (JBASR), vol. 1, no. 2, February 2013

[2] S. Azadi, A. Nikkerdar and M. Nouri, "Utilizing Azadi Controller (Positive Feedback) to Suppress the Vibrations of a DC Motor",Journal of Basic and Applied Scientific Research, pp. 10498-10507, 2012.

[3] S. Azadi, "Presenting an Adaptive Controller Based on Positive Feedback”in ICAFS, Prague, Czech., 2010, $27-$ 29August.

[4] S. Azadi, "Utilizing an Adaptive Controller (Azadi Controller) for Trajectory Planning of PUMA 560 Robot",in 2011 International Conference on Robotics and Cybernetics (ICCRC 2011), New Delhi,21-23 March 2011.

[5] S. Azadi, "Introducing a Simple Adaptive Controller (Azadi Controller) Based on Positive Feedbacks", Mianyang, CHINA, 2011 Chinese Control and Decision Conference (CCDC 2011), 23-25 May 2011. 
[6] S. A. Mazhari i S. Kumar, "Heuristic Search Algorithms for Tuning PUMA 560 Fuzzy PID Controller",International Journal of Computer Science, fall 2008

[7] R. Tanscheit and E. Lembessis, "On the behavior and tuning of a fuzzy rulebased self-organizing controller", in Mathematics of the analysis and design of process control, vol. 1, Amsterdam, Elsevier Science Publishers., 1992, p. 603-612.

[8] K. Ahn, D. Truong i Y. Soo, "Self tuning fuzzy PID control for hydraulic load simulator Automation and Systems" in IEEE International Conference on Control, Korea, 2007.

[9] E. Gonda, H. Miyata i M. Ohkita, "Self-tuning of fuzzy rules when learning data have a radically changing distribution", Electrical Engineering in Japan, vol. 144, nr 4, p. 63-74, 2003.

[10] F. Guely i P. \& Siarry, "Gradient descent method for optimizing various fuzzy rule bases", in Second IEEE international conference on fuzzy systems, 1993.

[11] P. Seihwan i H. Lee-kwang, "Designing fuzzy logic controllers by genetic algorithms considering their characteristics",in Congress on Evolutionary Computation, 2000.

[12] Y. C. Chiou i L. W. Lan, "Genetic fuzzy logic controller: an iterative evolution algorithm with new encoding method", Fuzzy Sets and Systems, nr 152, p. 617-635, 2005.

[13] R. Alcala, J. Benitez, J. Casillas, O. Cordon i R. Perez, "Fuzzy control of HVAC systems optimized by genetic algorithms", Applied Intelligence, vol. 2, nr 18, p. 155 177, 2003.

[14] F. Herrera, M. Lozano i J. L. Verdegay, "Tuning fuzzy logic controllers by genetic algorithms", International Journal of Approximate Reasoning, vol. 12, p. 299-315, 1995.

[15] A. Homaifar i E. McCormick, "Simultaneous design of membership functions and rule sets for fuzzy controllers using genetic algorithms", IEEE Transactions on Fuzzy Systems, vol. 3, nr 2, p. 129-139, 1995.

[16] H. Gurocak, "Genetic-algorithm-based method for tuning fuzzy logic controllers", Fuzzy Sets and Systems, vol. 108, nr 1, p. 39-47, Nov. 1999.
[17] H. Youssef, S. Sait i S. Khan, "Topology design of switched enterprise networks using a fuzzy simulated evolution algorithm", Eng. Appl. Artif. Intell., vol. 15, p. 327-340, 2002.

[18] L. Ingber i B. Rosen, "Genetic algorithms and very fast simulated reannealing", A comparison. Mathematical and Computer Modeling, vol. 16, nr 11, pp. 87-100, 1992.

[19] G. Liu i W. Yang, "Learning and tuning of fuzzy membership functions by simulated annealing algorithm", in IEEE Asia-Pacific conference on circuits and systems, 2000.

[20] W. Lei, K. Qi i W. Qidi, "Fuzzy logic based multioptimum programming in particle swarm optimization", w IEEE International Conference on Networking, Sensing and Control, Tucson, Arizona, USA, 2005.

[21] H.-M. Feng, "Self-generation fuzzy modeling systems through hierarchical recursive-based particle swarm optimization", Cybernet. Syst.: Int. J., nr 36, p. 623-639, 2005.

[22] Q. Kang, L. Wang i Q. Wu, "Research on fuzzy adaptive optimization strategy of particle swarm algorithm", Int. J. Inform. Technol., vol. 12, nr 3, p. 66-76, 2006.

[23] W. Pang, K.-p. Wang, C.-g. Zhou i L.-j. Dong, "Fuzzy discrete particle swarm optimization traveling salesman problem",w Fourth International Conference on Computer and Information Technology, 2004.

[24] R. Rajabioun, "Cuckoo Optimization Algorithm", Applied Soft Computing, pp. 5508-5518, 13 May 2011.

[25] Artur C. Guyton, “Textbook of Medical Physiology”, W. B. Saunders Company.

[26] Eric R. Kandel, James H. Schwartz, 2000, "Principles of Neural Science”, Elsevier Science Publishing Company.

[27] "Matlab Help", Mathworks, 2012.

[28] M. Mohammadzaheri, "Double-command fuzzy control of a nonlinear CSTR", in 3rd IEEE Conference on Industrial Electronics and Applications, 2008. ICIEA, Adelaide, 2008

[29] O. Katsuhico, "Modern Control Engineering". 